\title{
BIPOWER VARIATION FOR GAUSSIAN PROCESSES WITH STATIONARY INCREMENTS
}

\author{
OLE E. BARNDORFF-NIELSEN, ${ }^{*}$ University of Aarhus \\ JOSÉ MANUEL CORCUERA, ${ }^{* *}$ University of Barcelona \\ MARK PODOLSKIJ, ${ }^{* * *}$ University of Aarhus and CREATES \\ JEANNETTE H. C. WOERNER, ${ }^{* * * *}$ University of Göttingen
}

\begin{abstract}
Convergence in probability and central limit laws of bipower variation for Gaussian processes with stationary increments and for integrals with respect to such processes are derived. The main tools of the proofs are some recent powerful techniques of Wiener/Itô/Malliavin calculus for establishing limit laws, due to Nualart, Peccati, and others.

Keywords: Bipower variation; central limit theorem; chaos expansion; Gaussian process; multiple Wiener-Itô integrals
\end{abstract}

2000 Mathematics Subject Classification: Primary 60G15; 60F17

\section{Introduction}

The theory of bipower, and more generally multipower, variation has been developed out of questions raised in mathematical finance; for motivation, some first results, and applications, see [2], [3], [4], [5], [6], [8], [29], [30], [31], and [32]. It is natural, therefore, that initially the focus was on Brownian semimartingales, for which a rather complete and comprehensive theory is now available; cf. [10] (see also [9] and [21]). Extensions of the theory to Lévy processes and Itô semimartingales have been obtained, particularly by Jacod [18] (cf. also [5]), and applications to finance of such extensions are discussed in [20] and [28].

A further avenue of generalisation is to stochastic integrals with respect to Gaussian processes having stationary increments. Starting points in this direction are [7] and [14], which treated the power variation case, providing, in particular, a feasible central limit theorem for inference on the integrands in question. (As discussed in [7], an important early forerunner of that paper is a paper by Guyon and Leon [16], in which quadratic variation limit results for stationary Gaussian processes were derived.) The techniques used there, as well as in the present paper which considers the bipower case, come from very powerful recent results developed in the context of Wiener/Itô/Malliavin calculus, especially by Nualart, Peccati, and coauthors; see [24], [25], and [26] (cf. also [22]). (In fact, we believe that there are no other tools available that would allow derivation of the conclusions in the present paper.)

Received 30 April 2008; revision received 9 January 2009.

* Postal address: Department of Mathematical Sciences, University of Aarhus, Ny Munkegade, DK-8000 Aarhus C,

Denmark. Email address: oebn@imf.au.dk

** Postal address: Universitat de Barcelona, Gran Via de les Corts Catalanes 585, 08007 Barcelona, Spain.

Email address: jmcorcuera@ub.edu

*** Current address: Department of Mathematics, ETH Zürich, HG G32.2, 8092 Zürich, Switzerland.

Email address: mark.podolskij@math.ethz.ch

**** Postal address: Institut für Mathematische Stochastik, Maschmühlenweg 8-10, 37073 Göttingen, Germany.

Email address: woerner@math.uni-goettingen.de 
The structure of the paper is as follows. In Section 2 we list a number of background results needed in Appendix A for the proofs of the main results, which are presented in Sections 3 and 4. In Sections 3 and 4 we discuss limit laws of bipower variation for Gaussian processes with stationary increments and for integrals with respect to such processes, respectively. In Section 5 we conclude the paper. The proofs are given in Appendix A.

\section{Background}

In this section we review the basic concepts of the Wiener chaos expansion. In particular, we present a multiplication formula (Proposition 1) and a multivariate central limit theorem for a sequence of random variables which admit a chaos representation (Theorem 1). The latter is based on the theory for multiple stochastic integrals developed in [17], [24], and [26].

Consider a complete probability space $(\Omega, \mathcal{F}, \mathrm{P})$ and a Gaussian subspace $\mathscr{H}_{1}$ of $L^{2}(\Omega, \mathcal{F}, \mathrm{P})$ whose elements are zero-mean Gaussian random variables. Let $\mathbb{H}$ be a separable Hilbert space with scalar product denoted by $\langle\cdot, \cdot\rangle_{\mathbb{H}}$ and norm $\|\cdot\|_{\mathbb{H}}$. We will assume that there is an isometry,

$$
\begin{gathered}
W: \mathbb{H} \rightarrow \mathscr{H}_{1}, \\
h \mapsto W(h),
\end{gathered}
$$

in the sense that

$$
\mathrm{E}\left[W\left(h_{1}\right) W\left(h_{2}\right)\right]=\left\langle h_{1}, h_{2}\right\rangle_{\mathbb{H}} .
$$

It is easy to see that this map has to be linear.

For any $m \geq 2$, we denote by $\mathscr{H}_{m}$ the $m$ th Wiener chaos, that is, the closed subspace of $L^{2}(\Omega, \mathcal{F}, \mathrm{P})$ generated by the random variables $H_{m}(X)$, where $X \in \mathscr{H}_{1}, \mathrm{E}\left[X^{2}\right]=1$, and $H_{m}$ is the $m$ th Hermite polynomial, i.e. $H_{0}(x)=1$ and

$$
H_{m}(x)=(-1)^{m} \exp \left(\frac{x^{2}}{2}\right) \frac{\mathrm{d}^{m}}{\mathrm{~d} x^{m}}\left(\exp \left(-\frac{x^{2}}{2}\right)\right) .
$$

Suppose that $\mathbb{H}$ is infinite-dimensional, and let $\left\{e_{i}, i \geq 1\right\}$ be an orthonormal basis of $\mathbb{H}$. Denote by $\Lambda$ the set of all sequences $a=\left(a_{1}, a_{2}, \ldots\right), a_{i} \in \mathbb{N}$, such that all the terms, except a finite number of them, vanish. For $a \in \Lambda$, we set $a !=\prod_{i=1}^{\infty} a_{i} !$ and $|a|=\sum_{i=1}^{\infty} a_{i}$. For any multi-index $a \in \Lambda$, we define

$$
\Phi_{a}=\frac{1}{\sqrt{a !}} \prod_{i=1}^{\infty} H_{a_{i}}\left(W\left(e_{i}\right)\right) .
$$

The family of random variables $\left\{\Phi_{a}, a \in \Lambda\right\}$ is an orthonormal system. In fact,

$$
\mathrm{E}\left[\prod_{i=1}^{\infty} H_{a_{i}}\left(W\left(e_{i}\right)\right) \prod_{i=1}^{\infty} H_{b_{i}}\left(W\left(e_{i}\right)\right)\right]=\delta_{a b} a !
$$

where $\delta_{a b}$ denotes the Kronecker symbol. Moreover, $\left\{\Phi_{a}|a \in \Lambda| a \mid,=m\right\}$ is a complete orthonormal system in $\mathscr{H}_{m}$.

Let $a \in \Lambda$ with $|a|=m$. The mapping

$$
\begin{gathered}
I_{m}: \mathbb{H}^{\odot m} \rightarrow \mathscr{H}_{m}, \\
\bigotimes_{i=1}^{\infty} e_{i}^{\otimes a_{i}} \mapsto \prod_{i=1}^{\infty} H_{a_{i}}\left(W\left(e_{i}\right)\right),
\end{gathered}
$$


between the symmetric tensor product $\mathbb{H}^{\odot m}$, equipped with the norm $\sqrt{m !}\|\cdot\|_{\mathbb{H} \otimes m}$, and the $m$ th chaos $\mathcal{H}_{m}$ is a linear isometry. Here ' $\tilde{\otimes}$ ' denotes the symmetrization of the tensor product ' $\otimes$ ' and $I_{0}$ is the identity in $\mathbb{R}$.

For any $h=h_{1} \otimes \cdots \otimes h_{m}$ and $g=g_{1} \otimes \cdots \otimes g_{m} \in \mathbb{H}^{\otimes m}$, we define the $p$ th contraction of $h$ and $g$, denoted by $h \otimes_{p} g$, as the element of $\mathbb{H}^{\otimes 2(m-p)}$ given by

$$
h \otimes_{p} g=\left\langle h_{1}, g_{1}\right\rangle_{\mathbb{H}} \cdots\left\langle h_{p}, g_{p}\right\rangle_{\mathbb{H}} h_{p+1} \otimes \cdots \otimes h_{m} \otimes g_{p+1} \otimes \cdots \otimes g_{m} .
$$

This definition can be extended by linearity to any element of $\mathbb{H}^{\otimes m}$. The element $h \otimes_{p} g$ does not necessarily belong to $\mathbb{H}^{\odot(2 m-p)}$, even if $h$ and $g$ belong to $\mathbb{H}^{\odot m}$. We denote by $h \tilde{\otimes}_{p} g$ the symmetrization of $h \otimes_{p} g$.

Proposition 1. For any $h \in \mathbb{H}^{\otimes p}$ and $g \in \mathbb{H}^{\otimes q}$, we have

$$
I_{p}(h) I_{q}(g)=\sum_{r=0}^{p \wedge q} r !\left(\begin{array}{l}
p \\
r
\end{array}\right)\left(\begin{array}{l}
q \\
r
\end{array}\right) I_{p+q-2 r}\left(h \tilde{\otimes}_{r} g\right) .
$$

Proof. First, note that

$$
I_{1}\left(e_{i}\right)=W\left(e_{i}\right)
$$

Let $a \in \Lambda$ with $|a|=p$ and $q=1$. Owing to the linearity of $I_{p}$, it suffices to consider the case in which $h=\widetilde{\otimes}_{i=1}^{\infty} e_{i}^{\otimes a_{i}}$ and $g=e_{j}$. It holds that

$$
I_{p}\left(\varlimsup_{i=1}^{\infty} e_{i}^{\otimes a_{i}}\right) I_{1}\left(e_{j}\right)=\prod_{i=1}^{\infty} H_{a_{i}}\left(W\left(e_{i}\right)\right) W\left(e_{j}\right) .
$$

Assume that $j$ is an index such that $a_{j}=0$. Then

$$
\bigotimes_{i=1}^{\infty} e_{i}^{\otimes a_{i}} \tilde{\otimes}_{1} e_{j}=0
$$

and

so we have

$$
\prod_{i=1}^{\infty} H_{a_{i}}\left(W\left(e_{i}\right)\right) W\left(e_{j}\right)=I_{p+1}\left(\widetilde{\bigotimes}_{i=1}^{\infty} e_{i}^{\otimes a_{i}} \tilde{\otimes} e_{j}\right)
$$

$$
I_{p}\left(\widetilde{\bigotimes}_{i=1}^{\infty} e_{i}^{\otimes a_{i}}\right) I_{1}\left(e_{j}\right)=I_{p+1}\left(\widetilde{\bigotimes}_{i=1}^{\infty} e_{i}^{\otimes a_{i}} \tilde{\otimes} e_{j}\right)+p I_{p-1}\left(\widetilde{\bigotimes}_{i=1}^{\infty} e_{i}^{\otimes a_{i}} \tilde{\otimes}_{1} e_{j}\right) .
$$

Now assume that $a_{j} \neq 0$. Then we obtain the identity

$$
\widehat{\bigotimes}_{i=1}^{\infty} e_{i}^{\otimes a_{i}} \tilde{\otimes}_{1} e_{j}=\frac{a_{j}}{p} \underset{\bigotimes_{i=1}^{\infty}}{\otimes} e_{i}^{\otimes a_{i}^{\prime}}
$$

with $a_{i}^{\prime}=a_{i}$ if $i \neq j$ and $a_{j}^{\prime}=a_{j}-1$. Furthermore,

$$
\begin{aligned}
\prod_{i=1}^{\infty} H_{a_{i}}\left(W\left(e_{i}\right)\right) W\left(e_{j}\right) & =\prod_{i=1, i \neq j}^{\infty} H_{a_{i}}\left(W\left(e_{i}\right)\right)\left(H_{a_{j}+1}\left(W\left(e_{j}\right)\right)+a_{j} H_{a_{j}-1}\left(W\left(e_{j}\right)\right)\right) \\
& =I_{p+1}\left(\bigotimes_{i=1}^{\infty} e_{i}^{\otimes a_{i}} \tilde{\otimes} e_{j}\right)+p I_{p-1}\left(\widetilde{\bigotimes}_{i=1}^{\infty} e_{i}^{\otimes a_{i}} \tilde{\otimes}_{1} e_{j}\right),
\end{aligned}
$$


since the Hermite polynomials verify that

$$
H_{n+1}(x)=x H_{n}(x)-n H_{n-1}(x) .
$$

Hence, relationship (1) is true for $q=1$. The general formula follows by induction through the lines of the proof of Proposition 1.1.3 of [23].

Remark 1. Note that if we take $h=e_{i}^{\otimes p}$ and $g=e_{i}^{\otimes q}$ then we obtain the well-known identity

$$
H_{p}\left(W\left(e_{i}\right)\right) H_{q}\left(W\left(e_{i}\right)\right)=\sum_{r=0}^{p \wedge q} r !\left(\begin{array}{l}
p \\
r
\end{array}\right)\left(\begin{array}{l}
q \\
r
\end{array}\right) H_{p+q-2 r}\left(W\left(e_{i}\right)\right) .
$$

Now, let $g$ be the $\sigma$-field generated by the random variables $\{W(h) \mid h \in \mathbb{H}\}$. Any squareintegrable random variable $F \in L^{2}(\Omega, g, P)$ has a unique chaos decomposition

$$
F=\sum_{m=0}^{\infty} I_{m}\left(h_{m}\right)
$$

where $h_{m} \in \mathbb{H}^{\odot m}$ (see [23, p. 13] for more details).

Finally, we present a multivariate central limit theorem for sequences of functionals $F_{n} \in$ $L^{2}(\Omega, g, P)$.

Theorem 1. Consider a sequence of d-dimensional random vectors $F_{n}=\left(F_{n}^{1}, F_{n}^{2}, \ldots, F_{n}^{d}\right)$, such that $F_{n}^{k} \in L^{2}(\Omega, g, P)$ and

$$
F_{n}^{k}=\sum_{m=0}^{\infty} I_{m}\left(h_{m, n}^{k}\right)
$$

where $h_{m, n}^{k} \in \mathbb{H}^{\odot m}$. Assume that the following conditions hold.

(i) For any $k=1, \ldots, d$, we have

$$
\lim _{N \rightarrow \infty} \limsup _{n \rightarrow \infty} \sum_{m=N+1}^{\infty} m !\left\|h_{m, n}^{k}\right\|_{\mathbb{H}^{\otimes m}}^{2}=0 .
$$

(ii) For $k, l=1, \ldots, d$, we have

$$
\begin{gathered}
m ! \lim _{n \rightarrow \infty}\left\|h_{m, n}^{k}\right\|_{\mathbb{H} \otimes m}^{2}=\Sigma_{k k}^{m}, \\
m ! \lim _{n \rightarrow \infty}\left\langle h_{m, n}^{k}, h_{m, n}^{l}\right\rangle_{\mathbb{H} \otimes m}=\Sigma_{k l}^{m}, \quad k \neq l,
\end{gathered}
$$

and $\sum_{m=1}^{\infty} \Sigma^{m}=\Sigma \in \mathbb{R}^{d \times d}$.

(iii) For any $m \geq 1, k=1, \ldots, d$, and $r=1, \ldots, m-1$,

$$
\lim _{n \rightarrow \infty}\left\|h_{m, n}^{k} \otimes_{r} h_{m, n}^{k}\right\|_{\mathbb{H}^{\otimes 2(m-r)}}^{2}=0 .
$$

Then we have

$$
F_{n}-h_{0, n} \stackrel{\mathrm{D}}{\rightarrow} N_{d}(0, \Sigma) \text { as } n \rightarrow \infty,
$$

where ' $\stackrel{\mathrm{D}}{\rightarrow}$ ' denotes convergence in distribution, and, for any natural number $N$ and $k=1, \ldots, d$,

$$
\lim _{n \rightarrow \infty} \mathrm{E}\left[\left(\sum_{m=1}^{N} I_{m}\left(h_{m, n}^{k}\right)\right)^{4}\right]=3\left(\sum_{m=1}^{N} \Sigma_{k k}^{m}\right)^{2}
$$


Proof. Under conditions (ii) and (iii), the weak convergence (2) of the vector

$$
\left(I_{m_{1}}\left(h_{m_{1}, n}^{1}\right), I_{m_{2}}\left(h_{m_{2}, n}^{2}\right), \ldots, I_{m_{d}}\left(h_{m_{d}, n}^{d}\right)\right)
$$

is shown in [26] (moreover, these authors proved that (2) implies (3)). Under the additional condition (i), this result can be extended to general multivariate sequences $F_{n}$ with squareintegrable components (see [7]).

\section{Asymptotic theory for bipower variation of Gaussian processes with stationary increments}

We consider a Gaussian process $\left(G_{t}\right)_{t \geq 0}$, defined on a filtered complete probability space $\left(\Omega, \mathcal{F},\left(\mathcal{F}_{t}\right)_{t \geq 0}, \mathrm{P}\right)$, with centred and stationary increments. The variance function $R$ of the increments of $G$ is defined as

$$
R(t)=\mathrm{E}\left[\left|G_{s+t}-G_{s}\right|^{2}\right], \quad t \geq 0 .
$$

In this section we study the asymptotic behaviour of the bipower variation processes

$$
V(G ; p, q)_{t}^{n}=\frac{1}{n \tau_{n}^{p+q}} \sum_{i=1}^{[n t]}\left|\Delta_{i}^{n} G\right|^{p}\left|\Delta_{i+1}^{n} G\right|^{q}, \quad p, q \geq 0,
$$

where $\Delta_{i}^{n} G=G_{i / n}-G_{(i-1) / n}$ and $\tau_{n}^{2}=R(1 / n)=\mathrm{E}\left[\left|\Delta_{i}^{n} G\right|^{2}\right]$, using the multiplication formula (1) and the central limit theorem discussed in the previous section. For this purpose, we introduce the representation

$$
|x|^{p}=\sum_{m=0}^{\infty} a_{p, m} H_{m}(x),
$$

where the $H_{m}$ are Hermite polynomials as defined in Section 2.

In order to give a statement about the asymptotic behaviour of the bipower variation process $V(G ; p, q)_{t}^{n}$, we require the following assumptions on the variance function $R$ defined in (4), which were introduced by Guyon and Leon [16].

(A1) $R(t)=t^{\beta} L_{0}(t)$ for some $\beta \in(0,2)$ and some positive slowly varying (at 0$)$ function $L_{0}$, which is continuous on $(0, \infty)$.

(A2) $R^{\prime \prime}(t)=t^{\beta-2} L_{2}(t)$ for some slowly varying function $L_{2}$, which is continuous on $(0, \infty)$.

(A3) There exists a $b \in(0,1)$ with

$$
K=\limsup _{x \rightarrow 0} \sup _{y \in\left[x, x^{b}\right]}\left|\frac{L_{2}(y)}{L_{0}(x)}\right|<\infty .
$$

Recall that a function $L:(0, \infty) \rightarrow \mathbb{R}$ is called slowly varying at 0 when the identity

$$
\lim _{x \searrow 0} \frac{L(t x)}{L(x)}=1
$$

holds for any fixed $t>0$. Provided that $L$ is continuous on $(0, \infty)$, we have

$$
|L(x)| \leq C x^{-\alpha}, \quad x \in(0, T],
$$

for any $\alpha>0$ and any $T>0$ (where the constant $C>0$ depends on $\alpha$ and $T$ ). 
Finally, we introduce the correlation function of the increments of $G$, i.e.

$$
r_{n}(j)=\operatorname{cov}\left(\frac{\Delta_{1}^{n} G}{\tau_{n}}, \frac{\Delta_{1+j}^{n} G}{\tau_{n}}\right), \quad j \geq 0 .
$$

By the polarization identity, and due to the stationarity of the increments of $G$, we know that $r_{n}(0)=1$ and

$$
r_{n}(j)=\frac{R((j+1) / n)+R((j-1) / n)-2 R(j / n)}{2 R(1 / n)}, \quad j \geq 1 .
$$

We start with the weak law of large numbers for the sequence $V(G ; p, q)_{t}^{n}$. Throughout this paper, we write $Y^{n} \stackrel{\text { ucp }}{\longrightarrow} Y$ when $\sup _{t \in[0, T]}\left|Y_{t}^{n}-Y_{t}\right| \stackrel{\mathrm{P}}{\rightarrow} 0$ for any $T>0$, where $\stackrel{\text { }}{\rightarrow}$ ' denotes convergence in probability.

Theorem 2. Assume that conditions (A1)-(A3) are satisfied. Then we have

$$
\frac{V(G ; p, q)_{t}^{n}}{\rho_{p, q}^{(n)}} \stackrel{\mathrm{ucp}}{\longrightarrow} t
$$

where the quantity $\rho_{p, q}^{(n)}$ is given by

$$
\rho_{p, q}^{(n)}=\sum_{m=0}^{\infty} a_{p, m} a_{q, m} m ! r_{n}^{m}(1)
$$

Proof. See Appendix A.

Remark 2. Note that, by the orthogonality of Hermite polynomials and (5), the identity

$$
\rho_{p, q}^{(n)}=\mathrm{E}\left[\left|\frac{\Delta_{i}^{n} G}{\tau_{n}}\right|^{p}\left|\frac{\Delta_{i+1}^{n} G}{\tau_{n}}\right|^{q}\right]
$$

holds. Moreover, since the function $L_{0}$ is slowly varying at 0 , assumption (A1), (6), and (7) (and the dominated convergence theorem) imply that

$$
\rho_{p, q}=\lim _{n \rightarrow \infty} \rho_{p, q}^{(n)}=\sum_{m=0}^{\infty} a_{p, m} a_{q, m} m !\left(2^{\beta-1}-1\right)^{m}=\mathrm{E}\left[\left|B_{i}^{\beta / 2}-B_{i-1}^{\beta / 2}\right|^{p}\left|B_{i+1}^{\beta / 2}-B_{i}^{\beta / 2}\right|^{q}\right],
$$

where $B^{\beta / 2}$ is the fractional Brownian motion with Hurst parameter $\beta / 2$. Consequently, Theorem 2 yields the uniform convergence

$$
V(G ; p, q)_{t}^{n} \stackrel{\mathrm{ucp}}{\longrightarrow} \rho_{p, q} t .
$$

Next, we present the weak limit of the properly normalized sequence $V(G ; p, q)_{t}^{n}$. Note that the central limit theorem for bipower variation is valid under the same assumptions that are required to show the corresponding result for the power variation case (see [7]).

Theorem 3. Assume that conditions (A1)-(A3) hold and that $0<\beta<\frac{3}{2}$. Then we obtain the weak convergence (in the space $\mathscr{D}([0, T])^{2}$ equipped with the local uniform topology)

$$
\left(G_{t}, \sqrt{n}\left(\frac{V(G ; p, q)_{t}^{n}}{\rho_{p, q}^{(n)}}-t\right)\right) \stackrel{\mathrm{w}}{\rightarrow}\left(G_{t}, \frac{\sigma_{p, q}}{\rho_{p, q}} W_{t}\right),
$$

where $W$ is a Brownian motion that is defined on an extension of the filtered probability space 
$\left(\Omega, \mathcal{F},\left(\mathcal{F}_{t}\right)_{t \geq 0}, \mathrm{P}\right)$ and is independent of $\mathcal{F}$, and $\sigma_{p, q}^{2}$ is given by

$$
\sigma_{p, q}^{2}=\lim _{n \rightarrow \infty} n \operatorname{var}\left(V\left(B^{\beta / 2} ; p, q\right)_{1}^{n}\right),
$$

where $B^{\beta / 2}$ is the fractional Brownian motion with Hurst parameter $\beta / 2$.

Proof. See Appendix A.

Remark 3. In Theorem 3 the constant $\rho_{p, q}^{(n)}$ cannot be replaced by its limit $\rho_{p, q}$ defined in (8). This is due to the fact that the bias, $\sqrt{n}\left(\rho_{p, q}^{(n)}-\rho_{p, q}\right)$, can, in general, converge to $\infty$.

Remark 4. The finiteness of $\sigma_{p, q}^{2}$ (for $0<\beta<\frac{3}{2}$ ) is shown in (19) in Appendix A. Note that, owing to assumption (A1), the behaviour of the function $R$ near 0 is similar to that of the fractional Brownian motion with Hurst parameter $\beta / 2$. This is reflected in (10).

Remark 5. As for the power variation case (see [7]), we expect the (pointwise) limit of $\sqrt{n}\left(V(G ; p, q)_{t}^{n} / \rho_{p, q}^{(n)}-t\right)$ to be an element of the second Wiener chaos if $\frac{3}{2}<\beta<2$ (in particular, it is not normal). When $\beta=\frac{3}{2}$, both types of limits may appear. See [16] for a detailed discussion of different cases.

The proof of Theorem 3 relies on the methods developed in the previous section. In the first step we apply the multiplication formula (1) to obtain the chaos decomposition of the sequence $\sqrt{n}\left(V(G ; p, q)_{t}^{n} / \rho_{p, q}^{(n)}-t\right)$. Then we show the convergence of finite-dimensional distributions of the sequence given in (9). Finally, we prove the tightness condition.

Note that the weak convergence in (9) is equivalent to the stable convergence (in $\mathscr{D}([0, T])$ )

$$
\sqrt{n}\left(\frac{V(G ; p, q)_{t}^{n}}{\rho_{p, q}^{(n)}}-t\right) \stackrel{\mathcal{F}^{G}-s t}{\longrightarrow} \frac{\sigma_{p, q}}{\rho_{p, q}} W_{t},
$$

where $\mathcal{F}^{G}$ denotes the $\sigma$-algebra generated by process $G$ (see [1], [19, p. 512], or [27] for more details on stable convergence) and $Y^{n} \stackrel{\mathcal{F}^{G}-s t}{\longrightarrow} Y\left(Y^{n}\right.$ and $Y$ are stochastic processes) means that $Y^{n}$ converges $\mathcal{F}^{G}$-stably in law to $Y$. The latter result is crucial for proving a functional central limit theorem for the bipower variation of integral processes, which is presented below.

\section{Extensions to integral processes}

In this section we extend the limit theorems of the previous section to integral processes

$$
Z_{t}=\int_{0}^{t} u_{s} \mathrm{~d} G_{s}
$$

defined on the same probability space as $G$, where the stochastic integral is the pathwise Riemann-Stieltjes integral. Assumption (A1) implies that $G$ has finite $r$-variation for any $r>2 / \beta$ and, hence, by [34], the integral in (11) is well defined for any stochastic process $u$ of finite $q$-variation with $q<1 /(1-\beta / 2)$.

Example 1. Let us present some concrete examples of the process defined in (11).

(i) Consider two Hurst parameters $H, H^{*} \in(0,1)$ with $H+H^{*}>1$. If $u_{s}=f\left(B_{s}^{H^{*}}\right)$ for some locally Lipschitz function $f$ then the integral

$$
Z_{t}=\int_{0}^{t} u_{s} \mathrm{~d} B_{s}^{H}
$$

is well defined in the pathwise Riemann-Stieltjes sense (note that $\beta=2 H$ ). 
(ii) In [13] the authors used a process of the form

$$
w_{t}^{H^{*}}=\int_{0}^{t} \frac{(t-s)^{H^{*}-1 / 2}}{\Gamma\left(H^{*}+1 / 2\right)} \mathrm{d} W_{s}, \quad \frac{1}{2}<H^{*}<1,
$$

where $W$ is a Brownian motion, to model the log-volatility of asset prices. This process is a truncated version of the fractional Brownian motion $B^{H^{*}}$. In fact, they considered the log-volatility, $x$, as the solution of the stochastic differential equation

$$
\mathrm{d} x_{t}=-k x_{t} \mathrm{~d} t+\sigma \mathrm{d} w_{t}^{H^{*}}, \quad t \geq 0
$$

with $x(0)=0$ and $k>0$. Then the asset price, $Z$, can be written as

$$
Z_{t}=\int_{0}^{t} \exp \left(x_{s}\right) \mathrm{d} W_{s}^{*}, \quad t \geq 0,
$$

where $W^{*}$ is another Brownian motion, possibly correlated with $W$. In this example, $\beta=1$ and the process $u_{t}=\exp \left(x_{t}\right)$ is $\left(H^{*}-\varepsilon\right)$-Hölder continuous for any $0<\varepsilon<H^{*}$. So the process $Z$ can be defined as a pathwise integral. These models try to capture the long-memory effect observed in real data on volatility.

In this example we can also replace $W^{*}$ by a fractional Brownian motion $B^{H}$ as long as $H+H^{*}>1$. These kinds of processes appear when asset prices, not only the volatility, are modelled by diffusions driven by a fractional Brownian motion. See [15] for an alternative model, using a fractional Brownian motion, to the well-known Black-Scholes model. However, the use of the fractional Brownian motion for modelling prices in finance is controversial, because these processes are not semimartingales. Then, without any additional assumption on the trading operations, these models imply the possibility of arbitrage. An interesting discussion on this point can be found in [11].

Now we state the law of large numbers for the integral process which is valid under the same assumptions as in the power variation case.

Theorem 4. Assume that conditions (A1)-(A3) hold. Suppose that $u=\left\{u_{t}, t \in[0, T]\right\}$ is a stochastic process with finite $r$-variation, where $r<1 /(1-\beta / 2)$. Set

$$
Z_{t}=\int_{0}^{t} u_{s} \mathrm{~d} G_{s}
$$

Then, for $p, q>0$, we obtain

$$
V(Z ; p, q)_{t}^{n} \stackrel{\text { ucp }}{\longrightarrow} \rho_{p, q} \int_{0}^{t}\left|u_{s}\right|^{p+q} \mathrm{~d} s \quad \text { as } n \rightarrow \infty .
$$

Proof. See Appendix A.

Next we provide the weak limit theorem of the properly normalized bipower variation.

Theorem 5. Assume that conditions (A1)-(A3) hold. Suppose that $u=\left\{u_{t}, t \in[0, T]\right\}$ is an $\mathcal{F}^{G}$-measurable stochastic process with finite $r$-variation, where $r<1 /(1-\beta / 2)$, and is Hölder continuous of the order a with $a>\max (1 /(2(p \wedge 1)), 1 /(2(q \wedge 1)))$. Then we obtain, for $Z_{t}=\int_{0}^{t} u_{s} \mathrm{~d} G_{s}$ and $p, q>0$,

$$
\left(G_{t}, \sqrt{n}\left(\frac{V(Z ; p, q)_{t}^{n}}{\rho_{p, q}^{(n)}}-\int_{0}^{t}\left|u_{s}\right|^{p+q} \mathrm{~d} s\right)\right) \stackrel{\mathrm{w}}{\rightarrow}\left(G_{t}, \frac{\sigma_{p, q}}{\rho_{p, q}} \int_{0}^{t}\left|u_{s}\right|^{p+q} \mathrm{~d} W_{s}\right) \text { as } n \rightarrow \infty
$$


where the convergence is in $\mathscr{D}([0, T])^{2}$ equipped with the local uniform topology, and $W$ is a Brownian motion defined on an extension of the filtered probability space $\left(\Omega, \mathcal{F},\left(\mathcal{F}_{t}\right)_{t \geq 0}, \mathrm{P}\right)$ and is independent of $\mathcal{F}$.

Proof. See Appendix A.

Remark 6. Theorem 3 holds for the processes introduced in Example 1 if the measurability condition is satisfied, i.e. $H+H^{*}>1$ and $H^{*}>\max (1 /(2(p \wedge 1)), 1 /(2(q \wedge 1)))$.

Combining Theorems 4 and 5 we can derive a standard central limit theorem for the bipower variation.

Corollary 1. Under the assumption of Theorem 5, it holds that

$$
\frac{\sqrt{n}\left(V(Z ; p, q)_{t}^{n} / \rho_{p, q}^{(n)}-\int_{0}^{t}\left|u_{s}\right|^{p+q} \mathrm{~d} s\right)}{\sqrt{\left(V(Z ; 2 p, 2 q)_{t}^{n} / \rho_{2 p, 2 q}\right)\left(\sigma_{p, q}^{2} / \rho_{p, q}^{2}\right)}} \stackrel{\mathrm{D}}{\rightarrow} N(0,1) .
$$

Remark 7. Finally, we demonstrate some applications of the limiting results presented in Theorems 4 and 5.

(i) In practice, the realised ratio statistic defined as

$$
R_{t}^{n}=\frac{\mu_{1}^{-2} V(Z ; 1,1)_{t}^{n}}{V(Z ; 2,0)_{t}^{n}}
$$

where $\mu_{1}=\mathrm{E}|U|, U \sim N(0,1)$, turns out to be very informative about the properties of the (unobserved) path of the process $Z$. When $Z$ is an Itô semimartingale it is well known (see, e.g. [6]) that $R_{t}^{n}$ converges in probability and $p-\lim _{n \rightarrow \infty} R_{t}^{n} \leq 1$. Hence, the statistic $R_{t}^{n}$ can be used to test whether the underlying process $Z$ is a semimartingale or not (we refer the reader to [33], where a similar type of statistic is used to solve the same test problem).

When $G=B^{H}, H \in(0,1), R_{t}^{n}$ can be used to estimate the Hurst parameter $H$. By Theorem 4 we have

$$
R_{t}^{n}=\frac{\mu_{1}^{-2} V(Z ; 1,1)_{t}^{n}}{V(Z ; 2,0)_{t}^{n}} \stackrel{\text { ucp }}{\longrightarrow} \mu_{1}^{-2} \mathrm{E}\left[\left|B_{1}^{H}\right|\left|B_{2}^{H}-B_{1}^{H}\right|\right]=c_{H} \arcsin \left(c_{H}\right)+\sqrt{1-c_{H}^{2}}
$$

with $c_{H}=2^{2 H-1}-1$. The right-hand side is bijective as a function in $H \in(0,1)$. Consequently, a consistent estimator $\hat{H}$ of the Hurst parameter $H$ can be constructed by using the above convergence.

(ii) Another useful property of the bipower variation is its robustness to certain jump processes. Consider a pure-jump semimartingale $Y$ of the form

$$
Y_{t}=Y_{0}+B(h)_{t}+h *\left(\mu_{t}-v_{t}\right)+(x-h(x)) * \mu_{t}, \quad t \geq 0,
$$

where $B(h)$ is a predictable process of bounded variation, $h$ is a truncation function that behaves like $x$ at the origin, $\mu$ is a jump measure, and $v$ is its predictable compensator. Let $\gamma$ be the Blumenthal-Getoor index of $Y$. When $\gamma<1$, we additionally assume that $B(h)+(x-h(x)) * v=0$. Then Theorem 4 is robust to $Y$ if

$$
\frac{2}{\beta}>\gamma \quad \text { and } \quad \frac{2}{\beta}>\max (p, q),
$$


i.e. the statistics $V(Z ; p, q)_{t}^{n}$ and $V(Z+Y ; p, q)_{t}^{n}$ have the same limit in probability. This property can be deduced by the same methods as presented in [14]. Under the stronger conditions

$$
\frac{1}{\beta}>\gamma \quad \text { and } \quad \frac{2}{\beta}>p+q>\frac{\gamma}{2-\gamma \beta},
$$

Theorem 5 is also robust to the presence of the process $Y$ (see again [14] for more details).

\section{Conclusion}

In this paper we derived convergence in probability and stable central limit theorems for bipower variation of Gaussian processes with stationary increments and for associated integral processes. The corresponding asymptotic theory for multipower variation can be obtained similarly in a straightforward manner. Extensions of the results presented here to spatial and tempo-spatial settings would be of interest, as would simulation and empirical studies of how well the limit laws work in applications.

\section{Appendix A}

In the following we denote all constants which do not depend on $n$ by $C$.

Let $\mathscr{H}_{1}$ be the first Wiener chaos associated with the triangular array $\left(\Delta_{j}^{n} G / \tau_{n}\right)_{n \geq 1,1 \leq j \leq[n t]}$, i.e. the closed subspace of $L^{2}(\Omega, \mathcal{F}, \mathrm{P})$ generated by the random variables

$$
\left(\Delta_{j}^{n} G / \tau_{n}\right)_{n \geq 1,1 \leq j \leq[n t]} .
$$

Note that $\mathscr{H}_{1}$ can be seen as a separable Hilbert space with a scalar product induced by the covariance function of the process $\left(\Delta_{j}^{n} G / \tau_{n}\right)_{n \geq 1,1 \leq j \leq[n t]}$. This means that we can apply the theory of Section 2 to the canonical Hilbert space $\mathbb{H}=\mathscr{H}_{1}$. Denote by $\mathscr{H}_{m}$ the $m$ th Wiener chaos associated with the triangular array $\left(\Delta_{j}^{n} G / \tau_{n}\right)_{n \geq 1,1 \leq j \leq[n t]}$, and denote by $I_{m}$ the corresponding linear isometry between the symmetric tensor product $\mathscr{H}_{1}^{\odot m}$ (equipped with the norm $\sqrt{m !}\|\cdot\|_{\mathcal{H}_{1}^{\otimes m}}$ ) and the $m$ th Wiener chaos.

First, we present the chaos decomposition for the sequence $V(G ; p, q)_{t}^{n}-\rho_{p, q}^{(n)} t$.

Lemma 1. For any $t>0$, we obtain the decomposition

$$
V(G ; p, q)_{t}^{n}-\rho_{p, q}^{(n)} t=\sum_{m=2}^{\infty} I_{m}\left(\frac{1}{n} \sum_{i=1}^{[n t]} f_{i}^{m}\right)+O\left(n^{-1}\right),
$$

where the kernels $f_{i}^{m} \in \mathcal{H}_{1}^{\odot m}$ are given by

$$
f_{i}^{m}=\sum_{h=0}^{m} s_{h, m}^{(n)}\left(\frac{\Delta_{i}^{n} G}{\tau_{n}}\right)^{\otimes h} \tilde{\otimes}\left(\frac{\Delta_{i+1}^{n} G}{\tau_{n}}\right)^{\otimes m-h}
$$

(for simplicity we suppress the dependency of $f_{i}^{m}$ on $n$ ) with

$$
s_{h, m}^{(n)}=\sum_{l=0}^{\infty} a_{p, l+h} a_{q, l+m-h} l !\left(\begin{array}{c}
l+h \\
l
\end{array}\right)\left(\begin{array}{c}
l+m-h \\
l
\end{array}\right) r_{n}^{l}(1) .
$$


Moreover, it holds that

$$
\begin{aligned}
\left|s_{h, m}^{(n)}\right| & \leq \sum_{l=0}^{\infty}\left|a_{p, l+h}\right|\left|a_{q, l+m-h}\right| l !\left(\begin{array}{c}
l+h \\
l
\end{array}\right)\left(\begin{array}{c}
l+m-h \\
l
\end{array}\right)\left|r_{n}^{l}(1)\right| \\
& \leq C\left(\begin{array}{c}
m \\
h
\end{array}\right)\left(\frac{1}{\left|r_{n}(1)\right|\left(1-\left|r_{n}(1)\right|\right)}\right)^{m},
\end{aligned}
$$

where the constant $C$ does not depend on $n, m$, or $h$.

Proof. Using the multiplication formula (1) and the linearity of the mapping $I_{m}$, we obtain the representation

$$
\begin{aligned}
V(G ; p, q)_{t}^{n}= & \frac{1}{n} \sum_{i=1}^{[n t]} \sum_{m_{1}, m_{2}=0}^{\infty} a_{p, m_{1}} a_{q, m_{2}}\left(\sum_{l=0}^{m_{1} \wedge m_{2}} l !\left(\begin{array}{c}
m_{1} \\
l
\end{array}\right)\left(\begin{array}{c}
m_{2} \\
l
\end{array}\right)\right. \\
& \left.\times I_{m_{1}+m_{2}-2 l}\left(\left(\frac{\Delta_{i}^{n} G}{\tau_{n}}\right)^{\otimes m_{1}} \otimes_{l}\left(\frac{\Delta_{i+1}^{n} G}{\tau_{n}}\right)^{\otimes m_{2}}\right)\right) \\
= & \frac{1}{n} \sum_{i=1}^{[n t]} \sum_{m_{1}, m_{2}=0}^{\infty} a_{p, m_{1}} a_{q, m_{2}}\left(\sum_{l=0}^{m_{1} \wedge m_{2}} l !\left(\begin{array}{c}
m_{1} \\
l
\end{array}\right)\left(\begin{array}{c}
m_{2} \\
l
\end{array}\right) r_{n}^{l}(1)\right. \\
= & \sum_{m=0}^{\infty} I_{m}\left(\frac{1}{n} \sum_{i=1}^{[n t]} f_{i}^{m}\right) .
\end{aligned}
$$

Note that $a_{p, 2 m+1}=0$ for all $m \geq 0$ and $p \geq 0$, because the $H_{2 m+1}$ are odd functions. This implies the identity

$$
V(G ; p, q)_{t}^{n}-\rho_{p, q}^{(n)} t=\sum_{m=2}^{\infty} I_{m}\left(\frac{1}{n} \sum_{i=1}^{[n t]} f_{i}^{m}\right)+O\left(n^{-1}\right) .
$$

Now, observe the identity

$$
\operatorname{var}\left(\left|\frac{\Delta_{i}^{n} G}{\tau_{n}}\right|^{p}\right)=\sum_{l=2}^{\infty} a_{p, l}^{2} l !<\infty .
$$

From this we deduce that $a_{p, l}^{2} \leq C / l$ ! (for any fixed $p \geq 0$ ). Recall that $\left|r_{n}(1)\right|<1$, since $r_{n}$ is a correlation function of a process with stationary increments. Consequently, we obtain the inequality

$$
\begin{gathered}
\sum_{l=0}^{\infty}\left|a_{p, l+h}\right|\left|a_{q, l+m-h}\right| l !\left(\begin{array}{c}
l+h \\
1
\end{array}\right)\left(\begin{array}{c}
l+m-h \\
1
\end{array}\right)\left|r_{n}^{l}(1)\right| \\
\leq \frac{C}{h !(m-h) !} \sum_{l=0}^{\infty}(l+1) \cdots(l+m)\left|r_{n}(1)\right|^{l} \\
\quad=C\left(\begin{array}{c}
m \\
h
\end{array}\right)\left(\frac{1}{\left|r_{n}(1)\right|\left(1-\left|r_{n}(1)\right|\right)}\right)^{m},
\end{gathered}
$$

which completes the proof. 
Next, we present a lemma which has been shown in [7].

Lemma 2. Suppose that conditions (A1)-(A3) hold. Let $\epsilon>0$ with $\epsilon<2-\beta$. Define the sequence $r(j)$ by

$$
r(j)=(j-1)^{\beta+\epsilon-2}, \quad j \geq 2,
$$

and $r(0)=r(1)=1$. Then we obtain the following assertions.

(i) It holds that

$$
\frac{1}{n} \sum_{j=1}^{n} r^{2}(j) \rightarrow 0
$$

If, moreover, $\beta+\epsilon-2<-\frac{1}{2}$, it holds that

$$
\sum_{j=1}^{\infty} r^{2}(j)<\infty
$$

(ii) For any $0<\epsilon<2-\beta$ from (14), there exists a natural number $n_{0}(\epsilon)$ such that

$$
\left|r_{n}(j)\right| \leq C r(j), \quad j \geq 0,
$$

for all $n \geq n_{0}(\epsilon)$.

(iii) Set $\rho(0)=1$ and $\rho(j)=\frac{1}{2}\left((j-1)^{\beta}-2 j^{\beta}+(j+1)^{\beta}\right)$ for $j \geq 1$. Then it holds that

$$
r_{n}(j) \rightarrow \rho(j) \text { for any } j \geq 0 .
$$

Now we introduce two independent variables, $X_{i}^{n}(1)$ and $X_{i}^{n}(2) \sim N(0,1)$, that are given by

$$
X_{i}^{n}(1)=\frac{\Delta_{i}^{n} G}{\tau_{n}}, \quad X_{i}^{n}(2)=a_{n} \frac{\Delta_{i}^{n} G}{\tau_{n}}+b_{n} \frac{\Delta_{i+1}^{n} G}{\tau_{n}}
$$

with $b_{n}=\left(1-r_{n}^{2}(1)\right)^{-1 / 2}$ and $a_{n}=-\left(1 / r_{n}^{2}(1)-1\right)^{-1 / 2}$. Note that $a_{n}$ and $b_{n}$ converge, because $r_{n}(1) \rightarrow \rho(1)$ (see Lemma 2(iii)), and, consequently, they are bounded. It is clear that $f_{i}^{m}$ can be represented as

$$
f_{i}^{m}=\sum_{k_{l} \in\{1,2\}} c_{k_{1}, \ldots, k_{m}}^{n} X_{i}^{n}\left(k_{1}\right) \otimes \cdots \otimes X_{i}^{n}\left(k_{m}\right)
$$

for some constants $c_{k_{1}, \ldots, k_{m}}^{n}$. Note that all summands are orthogonal. We obtain

$$
\left\|f_{1}^{m}\right\|_{\mathcal{H}_{1}^{\otimes m}}^{2}=\sum_{k_{l} \in\{1,2\}}\left|c_{k_{1}, \ldots, k_{m}}^{n}\right|^{2}=: c_{m}^{n} .
$$

Let $c_{m}, c_{k_{1}, \ldots, k_{m}}$, and $\tilde{f}_{i}^{m}$ be analogues of $c_{m}^{n}, c_{k_{1}, \ldots, k_{m}}^{n}$, and $f_{i}^{m}$, respectively, which correspond to $G=B^{\beta / 2}$, where $B^{\beta / 2}$ denotes a fractional Brownian motion with Hurst parameter $\beta / 2$ (note that $c_{m}, c_{k_{1}, \ldots, k_{m}}$, and $\left\langle\tilde{f}_{i}^{m}, \tilde{f}_{j}^{m}\right\rangle_{\mathcal{H}_{1}^{\otimes m}}$ do not depend on $n$, because $B^{\beta / 2}$ is self-similar). Owing to $(13)$ and the convergence $r_{n}(j) \rightarrow \rho(j)$, we deduce that

$$
c_{k_{1}, \ldots, k_{m}}^{n} \rightarrow c_{k_{1}, \ldots, k_{m}}, \quad c_{m}^{n} \rightarrow c_{m}, \quad \text { and } \quad\left\langle f_{1}^{m}, f_{1+k}^{m}\right\rangle_{\mathcal{H}_{1}^{\otimes m}} \rightarrow\left\langle\tilde{f}_{1}^{m}, \tilde{f}_{1+k}^{m}\right\rangle_{\mathscr{H}_{1}^{\otimes m}},
$$


for all $m$ and $k$. Furthermore, we obtain (since $r_{n}(1) \rightarrow \rho(1)$ )

$$
\begin{gathered}
\operatorname{var}\left(\left|\frac{\Delta_{i}^{n} G}{\tau_{n}}\right|^{p}\left|\frac{\Delta_{i+1}^{n} G}{\tau_{n}}\right|^{q}\right)=\sum_{m=2}^{\infty} c_{m}^{n} m !<\infty, \\
\operatorname{var}\left(\left|\frac{\Delta_{i}^{n} G}{\tau_{n}}\right|^{p}\left|\frac{\Delta_{i+1}^{n} G}{\tau_{n}}\right|^{q}\right) \rightarrow \operatorname{var}\left(\left|B_{1}^{\beta / 2}\right|^{p}\left|B_{2}^{\beta / 2}-B_{1}^{\beta / 2}\right|^{q}\right)=\sum_{m=2}^{\infty} c_{m} m ! .
\end{gathered}
$$

Proof of Theorem 2. We first show the pointwise convergence $V(G ; p, q)_{t}^{n}-\rho_{p, q}^{(n)} t \stackrel{\mathrm{P}}{\rightarrow} 0$. Using expansion (12) and the stationarity of the increments of $G$, we obtain the identity $\operatorname{var}\left(V(G ; p, q)_{t}^{n}-\rho_{p, q}^{(n)} t\right)=\sum_{m=2}^{\infty} m !\left(\frac{[n t]}{n^{2}}\left\|f_{1}^{m}\right\|_{\mathcal{H}_{1}^{\otimes m}}^{2}+\frac{2}{n^{2}} \sum_{k=1}^{[n t]-1}([n t]-k)\left\langle f_{1}^{m}, f_{1+k}^{m}\right\rangle_{\mathcal{H}_{1}^{\otimes m}}\right)$.

On the other hand, we have (since $a_{n}$ and $b_{n}$ are bounded, $\left|r_{n}(j)\right| \leq C r(j)$ by Lemma 2(ii) and $r(j)$ is decreasing)

$$
\begin{aligned}
\left|\left\langle f_{1}^{m}, f_{1+k}^{m}\right\rangle_{\mathcal{H}_{1}^{\otimes m}}^{\otimes m}\right| & =\sum_{h_{l} \in\{1,2\}, g_{l} \in\{1,2\}} c_{h_{1}, \ldots, h_{m}}^{n} c_{g_{1}, \ldots, g_{m}}^{n} \prod_{l=1}^{m}\left\langle X_{i}^{n}\left(h_{l}\right), X_{i+k}^{n}\left(g_{l}\right)\right\rangle_{\mathcal{H}_{1}} \\
& \leq c_{m}^{n}(\operatorname{Cr}(k-1))^{m} .
\end{aligned}
$$

Since $\mathrm{E}\left[\left|X_{i}^{n}(1)\right|^{2}\right]=\mathrm{E}\left[\left|X_{i}^{n}(2)\right|^{2}\right]=1$, we also have

$$
\left|\left\langle f_{1}^{m}, f_{1+k}^{m}\right\rangle_{\mathscr{H}_{1}^{\otimes m}}\right| \leq c_{m}^{n} .
$$

Now, there exists a $Q$ with $C r(k-1)<1$ for all $k \geq Q$. It holds that

$$
\begin{aligned}
\frac{1}{n} \sum_{k=1}^{[n t]-1}([n t]-k)\left\langle f_{1}^{m}, f_{1+k}^{m}\right\rangle_{\mathscr{H}_{1}^{\otimes m}} & \leq Q c_{m}^{n}+\frac{1}{n}\left|\sum_{k=Q}^{[n t]-1}([n t]-k)\left\langle f_{1}^{m}, f_{1+k}^{m}\right\rangle_{\mathcal{H}_{1}^{\otimes m}}\right| \\
& \leq c_{m}^{n}\left(Q+\sum_{k=Q}^{[n t]-1}(\operatorname{Cr}(k-1))^{2}\right) \\
& \leq C c_{m}^{n} \sum_{k=1}^{[n t]-1} r^{2}(k) .
\end{aligned}
$$

Consequently, we deduce that

$$
\operatorname{var}\left(V(G ; p, q)_{t}^{n}-\rho_{p, q}^{(n)} t\right) \leq \frac{C}{n} \sum_{m=2}^{\infty} c_{m}^{n} m ! \sum_{k=1}^{[n t]-1} r^{2}(k) .
$$

By (15), (16), and Lemma 2(i), we obtain the pointwise convergence

$$
\frac{V(G ; p, q)_{t}^{n}}{\rho_{p, q}^{(n)}} \stackrel{\mathrm{P}}{\rightarrow} t
$$

The ucp convergence follows immediately, because $V(G ; p, q)_{t}^{n} / \rho_{p, q}^{(n)}$ is increasing in $t$ and the limit process $g(t)=t$ is continuous. 
Proof of Theorem 3. We divide the proof of Theorem 3 into two steps. In the first step we prove the convergence of the finite-dimensional distribution of the sequence

$$
\left(G_{t}, \sqrt{n}\left(\frac{V(G ; p, q)_{t}^{n}}{\rho_{p, q}^{(n)}}-t\right)\right) .
$$

Then we prove the tightness of this sequence.

Step 1. Define the vector $Y_{n}=\left(Y_{n}^{1}, \ldots, Y_{n}^{d}\right)^{\top}$ by

$$
Y_{n}^{k}=\frac{1}{\sqrt{n}} \sum_{i=\left[n a_{k}\right]+1}^{\left[n b_{k}\right]}\left(\left|\frac{\Delta_{i}^{n} G}{\tau_{n}}\right|^{p}\left|\frac{\Delta_{i+1}^{n} G}{\tau_{n}}\right|^{q}-\rho_{p, q}^{(n)}\right),
$$

where $\left(a_{k}, b_{k}\right], k=1, \ldots, d$, are disjoint intervals contained in [0,T]. Clearly, it suffices to prove that

$$
\left(G_{b_{k}}-G_{a_{k}}, Y_{n}^{k}\right)_{1 \leq k \leq d} \stackrel{\mathrm{D}}{\rightarrow}\left(G_{b_{k}}-G_{a_{k}}, \sigma_{p, q}\left(W_{b_{k}}-W_{a_{k}}\right)\right)_{1 \leq k \leq d},
$$

where $\sigma_{p, q}$ is given by (10) (because $\rho_{p, q}^{(n)} \rightarrow \rho_{p, q}$, where $\rho_{p, q}$ is given in (8)).

By Lemma 1 we obtain the representation

$$
Y_{n}^{k}=\sum_{m=2}^{\infty} I_{m}\left(\frac{1}{\sqrt{n}} \sum_{i=\left[n a_{k}\right]+1}^{\left[n b_{k}\right]} f_{i}^{m}\right)+O\left(n^{-1 / 2}\right) .
$$

Since

$$
\mathrm{E}\left[\left(G_{b_{k}}-G_{a_{k}}\right) Y_{n}^{l}\right]=0 \text { for any } 1 \leq k, l \leq d,
$$

it is sufficient to check the following conditions.

(i) For any $m \geq 1$ and $k=1, \ldots, d$, the limit

$$
\lim _{n \rightarrow \infty} m !\left\|\frac{1}{\sqrt{n}} \sum_{i=\left[n a_{k}\right]+1}^{\left[n b_{k}\right]} f_{i}^{m}\right\|_{\mathcal{H}_{1}^{\otimes m}}^{2}=\sigma_{p, q}^{2}(m, k)
$$

exists, $\sum_{m=2}^{\infty} \sigma_{p, q}^{2}(m, k)=\sigma_{p, q}^{2}\left(b_{k}-a_{k}\right)<\infty$, and

$$
\lim _{N \rightarrow \infty} \limsup _{n \rightarrow \infty} \sum_{m=N+1}^{\infty} m !\left\|\frac{1}{\sqrt{n}} \sum_{i=\left[n a_{k}\right]+1}^{\left[n b_{k}\right]} f_{i}^{m}\right\|_{\mathcal{H}_{1}^{\otimes m}}^{2}=0 .
$$

(ii) For any $m \geq 1$ and $k \neq h$,

$$
\lim _{n \rightarrow \infty}\left\langle\frac{1}{\sqrt{n}} \sum_{i=\left[n a_{k}\right]+1}^{\left[n b_{k}\right]} f_{i}^{m}, \frac{1}{\sqrt{n}} \sum_{i=\left[n a_{h}\right]+1}^{\left[n b_{h}\right]} f_{i}^{m}\right\rangle_{\mathcal{H}_{1}^{\otimes m}}=0 .
$$

(iii) For any $m \geq 1, k=1, \ldots, d$, and $1 \leq p \leq m-1$, we have

$$
\lim _{n \rightarrow \infty}\left\|\left(\frac{1}{\sqrt{n}} \sum_{i=\left[n a_{k}\right]+1}^{\left[n b_{k}\right]} f_{i}^{m}\right) \otimes_{p}\left(\frac{1}{\sqrt{n}} \sum_{i=\left[n a_{k}\right]+1}^{\left[n b_{k}\right]} f_{i}^{m}\right)\right\|_{\mathcal{H}_{1}^{\otimes 2(m-p)}}=0 .
$$


Under conditions (i)-(iii), we then obtain (by Theorem 1) the central limit theorem

$$
Y_{n} \stackrel{\mathrm{D}}{\rightarrow} N_{d}\left(0, \sigma_{p, q}^{2} \operatorname{diag}\left(b_{1}-a_{1}, \ldots, b_{d}-a_{d}\right)\right),
$$

where $\sigma_{p, q}^{2}$ is given by (10). Since the increments of the process $G$ are stationary, we will prove part (i) and (iii) only for $k=1, a_{1}=0$, and $b_{1}=1$.

(i) Recall (18):

$$
m !\left\|\frac{1}{\sqrt{n}} \sum_{i=1}^{n} f_{i}^{m}\right\|_{\mathcal{H}_{1}^{\otimes m}}^{2} \leq C m ! c_{m}^{n} \sum_{k=1}^{n-1} r^{2}(k) .
$$

Since $c_{m}^{n} \rightarrow c_{m}$ and $\sum_{k=1}^{\infty} r^{2}(k)<\infty$ (by Lemma 2(ii) with $0<\epsilon<\frac{3}{2}-\beta$ in the definition of $r$ (see (14))), we deduce by the dominated convergence theorem that

$$
\sigma_{p, q}^{2}(m, 1)=m !\left(\left\|\tilde{f}_{1}^{m}\right\|_{\mathcal{H}_{1}^{\otimes m}}^{2}+2 \sum_{k=1}^{\infty}\left\langle\tilde{f}_{1}^{m}, \tilde{f}_{1+k}^{m}\right\rangle_{\mathcal{H}_{1}^{\otimes m}}\right),
$$

where the kernels $\tilde{f}_{i}^{m}$ correspond to the case in which $G=B^{\beta / 2}$. Clearly,

$$
\lim _{n \rightarrow \infty} n \operatorname{var}\left(V\left(B^{\beta / 2} ; p, q\right)_{1}^{n}\right)=\sum_{m=2}^{\infty} \sigma_{p, q}^{2}(m, 1)=\sigma_{p, q}^{2}<\infty,
$$

because

$$
\sum_{m=2}^{\infty} \sigma_{p, q}^{2}(m, 1) \leq C \sum_{m=2}^{\infty} c_{m} m ! \sum_{k=1}^{\infty} r^{2}(k)<\infty
$$

owing to Lemma 2(i) and (16). On the other hand, we have

$$
\sum_{m=N+1}^{\infty} m !\left\|\frac{1}{\sqrt{n}} \sum_{i=1}^{n} f_{i}^{m}\right\|_{\mathcal{H}_{1}^{\otimes m}}^{2} \leq C \sum_{m=N+1}^{\infty} c_{m}^{n} m ! \sum_{k=1}^{\infty} r^{2}(k)
$$

and

$$
\lim _{n \rightarrow \infty} \sum_{m=N+1}^{\infty} c_{m}^{n} m !=\sum_{m=N+1}^{\infty} c_{m} m !
$$

for any fixed $N$ by (16) and $c_{m}^{n} \rightarrow c_{m}$. Since $\sum_{m=2}^{\infty} c_{m} m !<\infty$, we obtain

$$
\lim _{N \rightarrow \infty} \limsup _{n \rightarrow \infty} \sum_{m=N+1}^{\infty} m !\left\|\frac{1}{\sqrt{n}} \sum_{i=1}^{n} f_{i}^{m}\right\|_{\mathcal{H}_{1}^{\otimes m}}^{2}=0 .
$$

(ii) For any $1 \leq k, h \leq d$ with $b_{k} \leq a_{h}$, we have

$$
\left\langle\frac{1}{\sqrt{n}} \sum_{i=\left[n a_{k}\right]+1}^{\left[n b_{k}\right]} f_{i}^{m}, \frac{1}{\sqrt{n}} \sum_{i=\left[n a_{h}\right]+1}^{\left[n b_{h}\right]} f_{i}^{m}\right\rangle_{\mathcal{H}_{1}^{\otimes m}}=\frac{m !}{n} \sum_{j=\left[n a_{k}\right]+1}^{\left[n b_{k}\right]} \sum_{i=\left[n a_{h}\right]+1}^{\left[n b_{h}\right]}\left\langle f_{1}^{m}, f_{1+i-j}^{m}\right\rangle_{\mathcal{H}_{1}^{\otimes m}} .
$$

Assume, without loss of generality, that $a_{k}=0, b_{k}=a_{h}=1$, and $b_{h}=2$ (the case in which $b_{k}<a_{h}$ is much easier). By Lemma 2(ii) with $0<\epsilon<\frac{3}{2}-\beta$ in the definition of $r$ (see (14)), we obtain the approximation (by (17))

$$
\left|\left\langle\frac{1}{\sqrt{n}} \sum_{i=\left[n a_{k}\right]+1}^{\left[n b_{k}\right]} f_{i}^{m}, \frac{1}{\sqrt{n}} \sum_{i=\left[n a_{h}\right]+1}^{\left[n b_{h}\right]} f_{i}^{m}\right\rangle_{\mathscr{H}_{1}^{\otimes m}}\right| \leq C_{m}\left(\frac{1}{n} \sum_{j=1}^{n} j r^{m}(j)+\sum_{j=1}^{n-1} r^{m}(n+j)\right)
$$


for some constant $C_{m}>0$. It follows that $r^{m}(j) \leq(j-1)^{-1-\delta}$ for some $\delta>0$ and all $m, j \geq 2$. Hence, we obtain

$$
\left\langle\frac{1}{\sqrt{n}} \sum_{i=\left[n a_{k}\right]+1}^{\left[n b_{k}\right]} f_{i}^{m}, \frac{1}{\sqrt{n}} \sum_{i=\left[n a_{h}\right]+1}^{\left[n b_{h}\right]} f_{i}^{m}\right\rangle_{\mathcal{H}_{1}^{\otimes m}} \rightarrow 0 \quad \text { as } n \rightarrow \infty .
$$

(iii) Fix $1 \leq p \leq m-1$. Now, by Lemma 2(ii) (with $0<\epsilon<\frac{3}{2}-\beta$ in the definition of $r$ ) and (17), we deduce the inequality

$$
\begin{aligned}
& \left\|\left(\frac{1}{\sqrt{n}} \sum_{i=1}^{n} f_{i}^{m}\right) \tilde{\otimes}_{p}\left(\frac{1}{\sqrt{n}} \sum_{i=1}^{n} f_{i}^{m}\right)\right\|_{\mathcal{H}_{1}^{\otimes 2(m-p)}} \\
& \leq \frac{C_{m}}{n^{2}} \sum_{1 \leq j, l, h, k \leq n} r^{p}(|j-l|-1) r^{p}(|h-k|-1) \\
& \quad \times\left|\left\langle\left(\frac{\Delta_{j}^{n} G}{\tau_{n}}\right)^{\otimes(m-p)} \tilde{\otimes}\left(\frac{\Delta_{l}^{n} G}{\tau_{n}}\right)^{\otimes(m-p)},\left(\frac{\Delta_{h}^{n} G}{\tau_{n}}\right)^{\otimes(m-p)} \tilde{\otimes}\left(\frac{\Delta_{k}^{n} G}{\tau_{n}}\right)^{\otimes(m-p)}\right\rangle_{\mathcal{H}_{1}^{\otimes 2(m-p)} \mid}\right|,
\end{aligned}
$$

where ' $\sim$ ' denotes the symmetrization and $r(-1):=1$. Again applying Lemma 2(ii) and (17), we see that it suffices to prove that

$$
\begin{aligned}
& n^{-2} \sum_{1 \leq j, l, h, k \leq n} r^{p}(|j-l|-1) r^{p}(|h-k|-1) r^{\alpha}(|j-h|-1) r^{m-p-\alpha}(|l-h|-1) r^{m-p-\alpha} \\
& \quad \times(|j-k|-1) r^{\alpha}(|l-k|-1) \\
& \quad \rightarrow 0,
\end{aligned}
$$

where $0 \leq \alpha \leq m-p$. The latter term is smaller than

$$
n^{-1} \sum_{-1 \leq j, l, k \leq n-2} r^{p}(|j-l|) r^{p}(k) r^{\alpha}(j) r^{m-p-\alpha}(l) r^{m-p-\alpha}(|j-k|) r^{\alpha}(|l-k|) .
$$

Without any loss of generality, we can assume that $p=m-p=1$ and $\alpha=0$ or $\alpha=1$. For $\alpha=0$ and any $0<\varepsilon<1$, we obtain

$$
\begin{aligned}
& n^{-1} \sum_{-1 \leq j \leq n-2}\left(\sum_{-1 \leq l \leq n-2} r(|j-l|) r(l)\right)^{2} \\
& \leq n^{-1} \sum_{-1 \leq j \leq[n \varepsilon]}\left(\sum_{-1 \leq l \leq n-2} r(|j-l|) r(l)\right)^{2} \\
&+2 n^{-1} \sum_{[n \varepsilon]<j \leq n-2}\left(\sum_{-1 \leq l \leq[n \varepsilon / 2]} r(|j-l|) r(l)\right)^{2} \\
&+2 n^{-1} \sum_{[n \varepsilon]<j \leq n-2}\left(\sum_{[n \varepsilon / 2]<l \leq n-1} r(|j-l|) r(l)\right)^{2} \\
& \leq 2 \varepsilon\left(\sum_{-1 \leq l<n-2} r^{2}(l)\right)^{2}+6 \sum_{-1 \leq l<n-2} r^{2}(l) \sum_{[n \varepsilon / 2]<l<\infty} r^{2}(l),
\end{aligned}
$$


which converges to $2 \varepsilon\left(\sum_{-1 \leq l<\infty} r^{2}(l)\right)^{2}$ as $n \rightarrow \infty$ by Lemma 2(i). The desired result follows by letting $\varepsilon$ tend to 0 . Set

Step 2. Clearly, it suffices to show the tightness of the sequence $\sqrt{n}\left(V(G ; p, q)_{t}^{n}-\rho_{p, q}^{(n)} t\right)$.

$$
\sqrt{n}\left(V(G ; p, q)_{t}^{n}-\rho_{p, q}^{(n)} t\right)=\sum_{m=2}^{\infty} I_{m}\left(\frac{1}{\sqrt{n}} \sum_{i=1}^{[n t]} f_{i}^{m}\right)+O\left(n^{-1 / 2}\right)=: Z_{t}^{n}+O\left(n^{-1 / 2}\right)
$$

(where the approximation holds locally uniformly in $t$ ) and

$$
Z_{t}^{n, N}=\sum_{m=2}^{N} I_{m}\left(\frac{1}{\sqrt{n}} \sum_{i=1}^{[n t]} f_{i}^{m}\right)
$$

In step 1 we have proved that conditions (i)-(iii) of Theorem 1 are satisfied. Then, by (3) and the Cauchy-Schwarz inequality, we obtain the approximation

$$
\begin{aligned}
\mathrm{P}\left(\left|Z_{t}^{n, N}-Z_{t_{1}}^{n, N}\right| \geq \lambda,\left|Z_{t_{2}}^{n, N}-Z_{t}^{n, N}\right| \geq \lambda\right) \\
\quad \leq \frac{\mathrm{E}^{1 / 2}\left[\left|Z_{t}^{n, N}-Z_{t_{1}}^{n, N}\right|^{4}\right] \mathrm{E}^{1 / 2}\left[\left|Z_{t_{2}}^{n, N}-Z_{t}^{n, N}\right|^{4}\right]}{\lambda^{4}} \\
\quad \leq C \frac{\sigma_{p, q}^{4}\left([n t]-\left[n t_{1}\right]\right)\left(\left[n t_{2}\right]-[n t]\right)}{\lambda^{4}} \\
\quad \leq C \frac{\sigma_{p, q}^{4}\left(t_{2}-t_{1}\right)^{2}}{\lambda^{4}}
\end{aligned}
$$

for any $t_{1} \leq t \leq t_{2}$ and $\lambda>0$ (and the constant $C$ does not depend on $n$ ). On the other hand, (15) and (20) imply that

$$
\lim _{N \rightarrow \infty} \mathrm{E}\left[\left|Z_{t}^{n}-Z_{t}^{n, N}\right|^{2}\right]=0
$$

for any $n$ and any $t$. Using this, we conclude that

$$
\mathrm{P}\left(\left|Z_{t}^{n}-Z_{t_{1}}^{n}\right| \geq \lambda,\left|Z_{t_{2}}^{n}-Z_{t}^{n}\right| \geq \lambda\right) \leq C \frac{\sigma_{p, q}^{4}\left(t_{2}-t_{1}\right)^{2}}{\lambda^{4}}
$$

for any $t_{1} \leq t \leq t_{2}$ and $\lambda>0$, from which we deduce the tightness of the sequence $Z_{t}^{n}$ by Theorem 15.6 of [12]. This completes the proof of Theorem 3.

Proof of Theorem 4. The assertion of Theorem 4 follows from Theorem 2 by similar methods as presented in [7] (see the proof of Theorem 2 therein).

Proof of Theorem 5. The assertion of Theorem 5 follows from Theorem 3 by similar methods as presented in [7] (see the proof of Theorem 6 therein).

\section{Acknowledgements}

We would like to thank Shiying Si from the Department of Mathematics at the University of Tennessee for a very helpful remark on an earlier version of this paper. Ole E. BarndorffNielsen and Mark Podolskij acknowledge financial support from CREATES funded by the Danish National Research Foundation and from the Thiele Centre. The work of José Manuel Corcuera is supported by the MEC, grant number MTM2006-03211. 


\section{References}

[1] Aldous, D. J. and Eagleson, G. K. (1978). On mixing and stability of limit theorems. Ann. Prob. 6, 325-331.

[2] Barndorff-Nielsen, O. E. ANd Shephard, N. (2003). Realized power variation and stochastic volatility models. Bernoulli 9, 243-265.

[3] Barndorff-Nielsen, O. E. AND Shephard, N. (2004). Econometric analysis of realized covariation: high frequency covariance, regression, and correlation in financial economics. Econometrica 72, 885-925.

[4] Barndorff-Nielsen, O. E. And Shephard, N. (2004). Power and bipower variation with stochastic volatility and jumps (with discussion). J. Financial Econometrics 2, 1-48.

[5] Barndorff-Nielsen, O. E. And Shephard, N. (2006). Impact of jumps on returns and realised variances: econometric analysis of time-deformed Lévy processes. J. Econometrics 131, 217-252.

[6] Barndorff-Nielsen, O. E. AND Shephard, N. (2007). Variation, jumps, market frictions and high frequency data in financial econometrics. In Advances in Economics and Econometrics, eds R. Blundell et al., 9th World Congress, Cambridge University Press, pp. 328-372.

[7] Barndorff-Nielsen, O. E., Corcuera, J. M. and PodolskiJ, M. (2009). Power variation for Gaussian processes with stationary increments. To appear in Stoch. Process. Appl.

[8] Barndorff-Nielsen, O. E., Shephard, N. And Winkel, M. (2006). Limit theorems for multipower variation in the presence of jumps. Stoch. Process. Appl. 116, 796-806.

[9] Barndorff-Nielsen, O. E., Graversen, S. E., Jacod, J. And Shephard, N. (2006). Limit theorems for bipower variation in financial econometrics. Econometric Theory 22, 677-719.

[10] BARndorfF-Nielsen, O. E. et al. (2006). A central limit theorem for realised power and bipower variations of continuous semimartingales. In Stochastic Calculus to Mathematical Finance, eds Yu. Kabanov et al. Springer, Berlin, pp. 33-68.

[11] Bender, C., Sottinen, T. and Valkeyla, E. (2007). Arbitrage with fractional Brownian motion? Theory Stoch. Process. 13, 23-34.

[12] Billingsley, P. (1968). Convergence of Probability Measures. John Wiley, New York.

[13] Comte, F. And Renault, E. (1998). Long memory in continous-time stochastic volatility models. Math. Finance 8, 291-323.

[14] Corcuera, J. M., Nualart, D. and Woerner, J. H. C. (2006). Power variation of some integral fractional processes. Bernoulli 12, 713-735.

[15] Cultand, N. J., Kopp, P. E. And Willinger, W. (1995). Stock price returns and the Joseph effect: a fractional version of the Black-Scholes model. In Seminar of Stochastic Analysis, Random Fields and Applications (Ascona 1993; Progress Prob. 36), eds E. Bolthausen et al. Birkhäuser, Berlin, pp. 327-351.

[16] Guyon, L. ANd LeON, J. (1989). Convergence en loi des $H$-variations d'un processus gaussien stationnaire sur R. Ann. Inst. H. Poincaré Prob. Statist. 25, 265-282.

[17] Hu, Y. and Nualart, D. (2005). Renormalized self-intersection local time for fractional Brownian motion. Ann. Prob. 33, 948-983.

[18] JACOD, J. (2008). Asymptotic properties of realized power variations and related functionals of semimartingales. Stoch. Process. Appl. 118, 517-559.

[19] JaCoD, J. ANd Shiryaev, A. N. (2003). Limit Theorems for Stochastic Processes, 2nd edn. Springer, Berlin.

[20] JACOD, J. AND TODOROV, V. (2009). Testing for common arrival of jumps in discretely-observed multidimensional processes. To appear in Ann. Statist.

[21] Kinnebrock, S. And PodolskiJ, M. (2008). A note on the central limit theorem for bipower variation of general functions. Stoch. Process. Appl. 118, 1056-1070.

[22] Nourdin, I. and Peccati, G. (2009). Non-central convergence of multiple integrals. To appear in Ann. Prob.

[23] Nualart, D. (2006). The Malliavin Calculus and Related Topics, 2nd edn. Springer, Berlin.

[24] Nualart, D. and Peccati, G. (2005). Central limit theorems for sequences of multiple stochastic integrals. Ann. Prob. 33, 177-193.

[25] Nualart, D. and Ortiz-Latorre, S. (2008). Central limit theorems for multiple stochastic integrals and Malliavin calculus. Stoch. Process. Appl. 118, 614-628.

[26] Peccati, G. ANd Tudor, C. A. (2005). Gaussian limits for vector-valued multiple stochastic integrals. In Séminaire de Probabilités XXXVIII (Lecture Notes Math. 1857), eds M. Emery et al. Springer, Berlin, pp. 247262.

[27] RÉNYI, A. (1963). On stable sequences of events. Sankhyā A 25, 293-302.

[28] VeraART, A. (2009). Inference for the jump part of quadratic variation of Itô semimartingales. To appear in Econometric Theory.

[29] Woerner, J. H. C. (2003). Variational sums and power variation: a unifying approach to model selection and estimation in semimartingale models. Statist. Decisions 21, 47-68.

[30] Woerner, J. H. C. (2005). Estimation of integrated volatility in stochastic volatility models. Appl. Stoch. Models Business Industry 21, 27-44. 
[31] Woerner, J. H. C. (2006). Power and multipower variation: inference for high frequency data. In Stochastic Finance, eds A. N. Shiryaev et al. Springer, New York, pp. 343-364.

[32] Woerner, J. H. C. (2007). Inference in Lévy-type stochastic volatility models. Adv. Appl. Prob. 39, 531-549.

[33] Woerner, J. H. C. (2008). Volatility estimates for high frequency data: market microstructure noise versus fractional Brownian motion models. Working paper.

[34] Young, L. C. (1936). An inequality of the Hölder type, connected with Stieltjes integration. Acta Math. 67, 251-282. 